\title{
Conclusions des Journées Chevreul 2005 consacrées aux « Matières grasses végétales et industries agro-alimentaires »
}

\author{
Bernadette DELPLANQUE \\ Vice-présidente de l'AFECG \\ Laboratoire d'Endocrinologie de la Nutrition, \\ Université Paris Sud, Bat. 447 - \\ 91405 Orsay Cedex. \\ Fax: 0169005174 \\ <Bernadette.Delplanque@ibaic.u-psud.fr>
}

Le but de ces Journées Chevreul 2005 était de faire le point d'actualité et de provoquer des échanges entre producteurs, transformateurs et utilisateurs des matières grasses végétales en vue de répondre aux exigences réglementaires, nutritionnelles, qualitatives encadrant aujourd'hui les marchés.

En ces périodes où les réglementations évoluent et où les pressions écologiques et environnementales s'intensifient, ces deux jours ont vraiment été au cœur de l'actualité en présentant les développements technologiques ainsi que les besoins et les contraintes des industries agroalimentaires (IAA).

Au cours de la première journée, la succession des divers exposés a bien montré la complexité des éléments de traçabilité, de sécurité alimentaire, des exigences réglementaires ainsi que la lourdeur du cahier des charges (Pierre-Yves Vigneron, Lesieur RD).

Tous ont bien insisté sur l'importance des mises au point techniques nécessaires aux nouvelles demandes réglementaires, qui entraînent parfois même une remise en cause totale des techniques utilisées. Un gros travail a été fait dans ce sens par l'ensemble des industriels présents. Je voudrais relever deux points :

- L'évolution en baisse du marché des huiles et margarines, associée à une modification de consommation, puisqu'en quarante ans l'arachide a été remplacée par le tournesol, avec une montée en puissance de l'olive qui semble se stabiliser. À noter un grand absent, le colza, qui devrait accentuer sa percée très fortement et très rapidement.

- Le deuxième point qui m'est apparu inquiétant à première vue, c'est la perception de la nutrition, des graisses et lipides qu'en a le consommateur, perception « révélée » par des sondages, rapportés par Serge Michels. Évidemment, le consommateur n'est pas toujours à même de connaître la chimie, la biochimie des lipides ou d'être un expert en physiologie humaine pour faire ses propres choix nutritionnels. Cependant, si le consommateur reste un homme de bon sens, il semble parfois nécessaire de l' « éclairer », ce que font généralement les services marketing des sociétés agroalimentaires. II faut pourtant veiller à limiter les excès ou les oublis. II ne s'agit pas spécifiquement de la publicité qui est généralement très (et de plus en plus) réglementée et contrôlée, mais plutôt de certains invités d'émissions (télévisées par exemple), qui se permettent des allégations ou les amalgames plus que douteux servant leurs propres intérêts et qui sont là très difficiles à contrôler ou à rectifier a posteriori.

II reste à espérer que la nutrition, ou tout au moins ses bases, apportant la notion de besoin et d'équilibre, soit inscrite au programme des écoles et ce, dès le plus jeune âge: le programme Fleurbaix-Laventie-Ville-Santé en est I'illustration et devrait en être la justification (Education strategies: could children educate their parents? A Basdevant, D Boute, JM Borys International Journal of Obesity, 1999).

La deuxième journée a débuté par un instant d'émotion marqué par la remise de la Médaille Chevreul au Professeur Michel Parmentier qui nous a fait un remarquable exposé rapporté en détail dans ce dossier : Les membranes en lipotransformation : bilan, résultats, perspectives. Je retiendrai une de ses diapositives comme étant la meilleure illustration de l'intérêt de cette journée : "Toute amélioration se fait sous la pression d'évolution, de la qualité industrielle, de la consommation et de l'environnement, ce qui entraîne des progrès qui restent toujours à atteindre. » Cette phrase pourrait être ajoutée aux statuts de I'AFECG : en tout cas, elle en illustre bien les objectifs.

La grande réactivité des industriels à cette évolution et aux recommandations qui en découlent est un fait constant, un élément de survie très certainement, et elle est source de qualité et de sécurité. Ceci a été bien démontré par les exposés présentés au cours de ces journées: deux exemples :

- À propos des trans et des recommandations récentes de l'Afssa, il faut noter plusieurs années d'anticipation chez les huiliers et margariniers européens sur la réduction des trans, alors que les Américains en sont encore loin et nous envient (pour certains) d'avoir su mettre en place ces modifications visant une plus grande qualité nutritionnelle.

- Le deuxième exemple a été relayé par Gérard Pascal lors de son exposé sur les OGM. l'évolution des OGM vient de marquer une pause également sous différentes pressions : refus du consommateur européen d'une part et limites technologiques, qui ont conduit à une revalorisation de l'existant. Ainsi, il a montré qu'il existait un redéploiement vers I'utilisation des nouvelles technologies pour un retour vers des sélections végétales plus classiques, plutôt que vers un élargissement des nouvelles formules OGM.

On peut considérer que ces journées ont atteint leur objectif et démontré combien étaient importantes les interactions à tous les niveaux de l'agriculture à l'industrie agroalimentaire, jusqu'au consommateur qui a besoin de préserver ou retrouver une alimentation saine et équilibrée.

Enfin, le prix de Thèse AFECG a été remis à Anne Morise qui a présenté brillamment les résultats de son travail réalisé sous la direction du Dr Dominique Hermier et dont le sujet: «Effet de la dose d'acide alpha-linolénique alimentaire sur le métabolisme lipidique» démontre l'intérêt du précurseur végétal des oméga-3.

Pour conclure, un grand merci à Anne Daumas et Anne Rossignol-Castera pour avoir programmé et organisé ces journées, aux différents intervenants pour la très grande qualité de leurs exposés, ainsi qu'à tous les participants. 